\title{
Single-molecule force spectroscopy: Practical limitations beyond Bell's model
}

\author{
Sebastian Getfert, Mykhaylo Evstigneev, and Peter Reimann \\ Universität Bielefeld, Fakultät für Physik, 33615 Bielefeld, Germany
}

\begin{abstract}
Single-molecule force spectroscopy experiments, as well as a number of other physical systems, are governed by thermally activated transitions out of a metastable state under the action of a steadily increasing external force. The main observable in such experiments is the distribution of the forces, at which the escape events occur. The challenge in interpreting the experimental data is to relate them to the microscopic system properties. We work out a maximum likelihood approach and show that it is the optimal method to tackle this problem. When fitting actual experimental data it is unavoidable to assume some functional form for the force-dependent escape rate. We consider a quite general and common such functional form and demonstrate by means of data from a realistic computer experiment that the maximum number of fit parameters that can be determined reliably is three. They are related to the force-free escape rate and the position and height of the activation barrier. Furthermore, the results for the first two of these fit parameters show little dependence on the assumption about the manner in which the barrier decreases with the applied force, while the last one, the barrier height in the absence of force, depends strongly on this assumption.
\end{abstract}

PACS numbers: 82.37.Np, 33.15.Fm, 02.50.-r

\section{INTRODUCTION}

A quite remarkable experimental achievement of the last decade is the direct observation of chemical dissociation at the single-molecule level by applying timedependent external forces on the pico-Newton scale. This technique is called dynamic force spectroscopy or singlemolecule force spectroscopy and reviewed e.g. in [1, 2]. It allows to extract kinetic constants and energy landscape parameters of various interactions like antibodyantigen recognition [3] or protein-DNA interactions [4]. Also the dynamics of various other experimental systems are governed by thermally activated transitions out of a metastable state over a potential barrier, whose height decreases in time due to a steadily increasing external force. Examples include the polarization reversal in nanomagnets [5], friction at the atomic scale [6, 7, 8], and phase jumps in Josephson junctions [9, 10]. In all these cases, the knowledge of the force-dependent rate out of the metastable state can be exploited to characterize the system studied. The main theme of the present work is how to perform such a characterization in the most optimal way and to point out the limitations even of such an optimized procedure under practical conditions.

A typical experimental setup is schematically sketched in Fig. 1: the single chemical bond of interest, e.g. in a ligand-receptor complex, is connected via two linker molecules with the tip of an AFM (atomic force microscope) cantilever (or some other micromechanical tool) and a piezoelectric element. The latter is employed for "pulling down" the attached linker molecule at some constant velocity, leading to an elastic reaction force of the cantilever, determined from the deflection of a laser beam. The main quantity of interest is the magnitude of the force at the moment when the bond breaks.

The theoretical interpretation of the observed rupture forces is a non-trivial task for the following reasons. Upon repeating the same experiment with the same pulling ve-

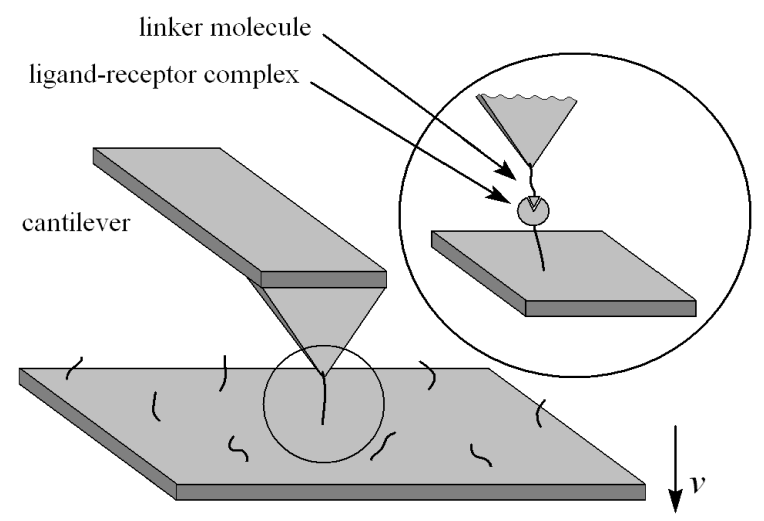

FIG. 1: (Color online) Schematic illustration of a singlemolecule force spectroscopy experiment. Receptor and ligand are connected via suitable linkers to the surface and the AFM tip, respectively. The distance of the tip form the surface can be controlled with a piezoelectric element (not shown). When pulled down at constant speed an (approximately linearly) increasing force acts on the bond which can be measured through the deflection of the cantilever.

locity, the rupture forces are found to be distributed over a wide range, contrary to what one would naively expect for a purely mechanical breaking of a compound object at some fixed, "critical" strain force. A further theoretical challenge represents the experimental finding that for different pulling velocities different such distributions are obtained. Hence, neither a single rupture event nor the average rupture force at any fixed pulling velocity can serve as a meaningful characteristics of a given chemical bond strength. Major steps in solving the puzzle are due to Bell [11] and to Evans and Ritchie [12], recognizing that a forced bond rup- 


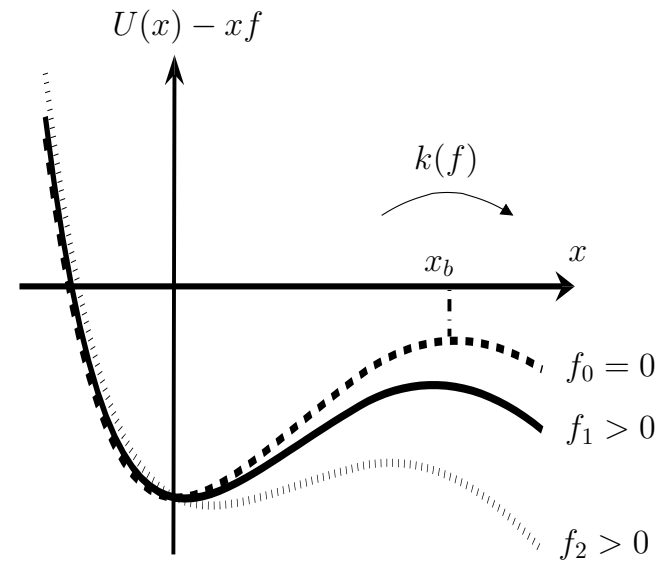

FIG. 2: Schematic illustration of the total relevant potential energy landscape $U(x)-x f$ of a receptor-ligand-bond as function of the reaction coordinate $x$ without and with externally applied bias forces $f_{1}<f_{2}$. For low forces the main effect is that the energy barrier $E_{\mathrm{b}}(f)$ is lowered by an amount $\Delta E_{\mathrm{b}} \simeq x_{\mathrm{b}} f$, where $x_{\mathrm{b}}$ is the distance between potential well and barrier at zero force. For larger forces this distance decreases.

ture event is a thermally activated decay of a metastable state that can be described within the general framework of Kramers reaction rate theory [13]. Subsequently, their basic theoretical approach has been extended and refined in several important directions, see e.g. Refs. [1, 2, 14, 15, 16, 17, 18, 19, 20, 21, 22, 23, 24, 25, 26, 27.

Following Evans and Ritchie [1, 12], a single-molecule dissociation process is viewed as thermally activated escape of a reaction coordinate $x$ over a potential barrier, see Fig. 2. Given the one-dimensional potential landscape along the reaction coordinate, the dissociation rate $k(f)$ for an instantaneous force $f$ (projected onto the direction of the reaction coordinate) can be written according to Kramers reaction-rate theory [13] in the form

$$
k(f)=\omega(f) \exp \left(-E_{b}(f) / k_{B} T\right),
$$

where the pre-exponential factor $\omega(f)$ has the intuitive meaning of an "attempt frequency" and the exponentially leading Boltzmann-Arrhenius factor contains the relevant potential barrier $E_{b}(f)$ against escape, Boltzmann's constant $k_{B}$, and the temperature $T$.

In dynamic force spectroscopy experiments, the rate at which the force increases is much slower than all other relevant molecular relaxation processes. Due to this separation of time scales [13], the reaction kinetics can be very accurately approximated by the following first-order differential equation

$$
\frac{d n(t)}{d t}=-k(f(t)) n(t)
$$

where $n(t)$ denotes the survival probability of the bond up to time $t$ and $k(f(t))$ is the accompanying dissociation rate (11) at an instantaneous external force $f(t)$.
Ideally, one would wish to use the experimentally established force-dependent escape rate $k(f)$ to reconstruct the potential landscape, i.e. potential energy vs. the reaction coordinate, cf. Fig. 2. However, in view of the fact that the escape rate depends on the energy difference at two force-dependent extrema, this problem does not have a unique solution [28]. Therefore, one may start with some model energy landscape, and try to deduce its global features, such as barrier height in the absence of the force and dissociation length (the distance between potential well and barrier without bias force, cf. Fig. (2). This introduces some specific functional form for the escape rate (11) involving several parameters, which are then determined by fitting the experimental data. In the present work, we describe the application of the maximum likelihood approach [29, 30] as a tool to deduce the model parameters. We show that this method is superior to any other approach that may be used for this purpose.

After introducing the method, we discuss its application to determine the parameters of a commonly used Ansatz for the rate due to Bell, henceforth called Bell's model [11, 12]. This approximation assumes that the force is sufficiently small, so that the force-dependent barrier height decreases linearly with the force, the proportionality constant $x_{\mathrm{b}}$ being the dissociation length (see Fig. 2),

$$
E_{\mathrm{b}}(f)=E_{\mathrm{b}}(0)-x_{\mathrm{b}} f
$$

The two fit parameters used in this approach are the escape rate at zero force, $k_{0}=\omega(0) e^{-E_{\mathrm{b}}(0) / k_{\mathrm{B}} T}$, and the dissociation length, $x_{\mathrm{b}}$. Within Bell's model, it is impossible to uncouple the intrinsic time and energy scales of the system, because multiplication of $\omega(0)$ by an arbitrary constant and addition of $k_{\mathrm{B}} T$ times the logarithm of that constant to $E_{\mathrm{b}}(0)$ leaves the force-free rate value, $k_{0}$, and hence the statistics of escape events, the same.

One of the central problems of the present work is gaining more information about the system than Bell's model allows. Obviously, in order to make it possible, one needs to use a functional form of the escape rate $k(f)$ involving more fit parameters than in Bell's Ansatz. Indeed, it has been suggested [6, 77, 8, 25, 26, 30] that one can actually estimate the value of the force-free activation barrier $E_{\mathrm{b}}(0)$ if one makes a more realistic assumption about how the barrier decreases from this value with increasing force; the linear decrease (3) is replaced with a non-linear potential. Then, the force-dependent rate involves more than two fit parameters, the force-free barrier height $E_{\mathrm{b}}(0)$ being one of them.

It is intuitively clear that the more rate parameters one has, the better one can characterize the system of interest. On the other hand, if the number of fit parameters is too large, then not all of them may be determined sufficiently accurately from the experimental data. Therefore, a relevant question is: what is the maximal number of rate parameters, which one can establish reliably? For a quite common and general parametrization of the potential we show that the highest number 
of model parameters one can determine from fitting the experimental data is three, while inclusion of additional parameters into the theory does not improve the quality of the fit. These three parameters are related to the forcefree escape rate, the height of the activation barrier, and the dissociation length. Furthermore, we show that the resulting fit values of these parameters strongly depend on the assumption concerning the manner in which the activation barrier decreases with the applied force. In other words, one cannot determine them uniquely without having this information.

\section{THEORETICAL MODELING OF BOND RUPTURE}

For a quantitative analysis of dynamic force spectroscopy experiments one usually assumes that the force $f(t)$ increases linearly,

$$
f(t)=\kappa v t
$$

where $v$ is the (constant) pulling velocity, and $\kappa$ the relevant total elasticity of cantilever, linker molecules, receptor and ligand, cf. Fig. 1. Using the fact that the extension $s$ of the complex at time $t$ is $s=v t$ we see that (4) is equivalent to the assumption of a linear forceextension characteristics.

Combining Eqs. (2) and (4), we arrive at the central experimental quantity, namely the probability density of escape events at the force value $f$ for a given velocity $v$ and a given set of parameters $\boldsymbol{\mu}$ characterizing the escape rate $k(f)$ :

$$
\begin{aligned}
& p_{1}(f \mid \boldsymbol{\mu}, v)=-\frac{d n(f)}{d f}=\frac{k(f)}{\kappa v} e^{-g(f) / \kappa v}, \\
& g(f):=\int_{0}^{f} d f^{\prime} k\left(f^{\prime}\right) .
\end{aligned}
$$

For later convenience, the rupture force distribution (5) is written as a conditional probability, conditioned on the values of the model parameters $\boldsymbol{\mu}$ and the pulling velocity $v$. While, in practice, the latter is directly accessible from the measurement, the model parameters $\boldsymbol{\mu}$ have to be inferred from the distribution of rupture forces.

It is possible to evaluate the integral (6) numerically for any functional form of the rate $k(f)$. However, the exponentially increasing character of this function, see Eq. (1), allows one to derive a very efficient analytical approximation for the integral. Namely, in view of Eq. (11), the main contribution to the integral $g(f)$ comes from the $f^{\prime}$-region just below $f$. This allows one to expand $\ln k\left(f^{\prime}\right)$ in the vicinity of the upper limit of integration $f$ to the first order [17]:

$$
\begin{aligned}
& k\left(f^{\prime}\right)=k(f) e^{\lambda_{1}(f)\left(f^{\prime}-f\right)}, \\
& \lambda_{n}(f):=(-1)^{n-1} \frac{d^{n} \ln k(f)}{d f^{n}} .
\end{aligned}
$$

With this approximation, the integral in (6) is given by

$$
g(f) \approx g_{1}(f)=\frac{k(f)}{\lambda_{1}(f)}\left(1-e^{-\lambda_{1}(f) f}\right) .
$$

This simple approximate formula may be sufficient for most practical purposes. However, if the deviations of $\ln k\left(f^{\prime}\right)$ from linearity are important, one can use the second-order approximation for this function near $f^{\prime}=f$ :

$$
k\left(f^{\prime}\right)=k(f) e^{\lambda_{1}(f)\left(f^{\prime}-f\right)-\lambda_{2}(f)\left(f^{\prime}-f\right)^{2} / 2},
$$

allowing one to evaluate the rate integral in (6) as

$$
\begin{aligned}
& g(f) \approx g_{2}(f)=k(f) e^{\lambda_{1}(f)^{2} /\left[2 \lambda_{2}(f)\right]} \sqrt{\frac{\pi}{2 \lambda_{2}(f)}} \\
& {\left[\operatorname{erf}\left(\sqrt{\frac{\lambda_{2}(f)}{2}}\left(f+\frac{\lambda_{1}(f)}{\lambda_{2}(f)}\right)\right)-\operatorname{erf}\left(\frac{\lambda_{1}(f)}{\sqrt{2 \lambda_{2}(f)}}\right)\right],}
\end{aligned}
$$

where $\operatorname{erf}(x):=2 \pi^{-1 / 2} \int_{0}^{x} d y e^{-y^{2}}$ is the error function. Additional numerical analysis has shown that the inaccuracy of this expression is smaller than $1 \%$ for all reasonable functional choices $k(f)$ which we have checked.

\section{PARAMETER ESTIMATION: PROPERTIES OF THE MAXIMUM LIKELIHOOD ESTIMATOR}

Let us assume that a specific model and thus the particular form of the probability density $p_{1}$ in (5) can be considered as given. Then the remaining task is to estimate the model parameters $\boldsymbol{\mu}$ from a given set of $N$ rupture forces $\mathbf{f}=\left\{f_{i}\right\}_{i=1}^{N}$ and pulling velocities $\mathbf{v}=\left\{v_{i}\right\}_{i=1}^{N}$. There exist different "recipes" for doing this, called estimators. Each of them can be formally represented by some function $\tilde{\boldsymbol{\mu}}(\mathbf{f})$, indicating that the estimate, being a function of the random variables $\mathbf{f}$, is a random variable itself. Now, the main question is: what is the optimal estimate of the model parameters that can be extracted from the given set of $N$ rupture forces? Stated differently: which recipe yields estimates $\tilde{\boldsymbol{\mu}}(\mathbf{f})$ of the model parameters which are on average over many data sets $\mathbf{f}$ closest to the "true" model parameters?

In this section we discuss some properties of estimators and show that under realistic experimental conditions, given in single-molecule pulling experiments, no estimator $\tilde{\boldsymbol{\mu}}(\mathbf{f})$ outperforms the maximum likelihood estimator. The reader who is not interested in the mathematical details may skip the subsequent subsections A-C and immediately proceed to subsection $\mathrm{D}$, where we summarize the main steps which are necessary for a practical application.

\section{A. Maximum Likelihood Estimator}

Our starting point is the probability $p$ to observe a given set of $N$ rupture forces $\mathbf{f}=\left\{f_{i}\right\}_{i=1}^{N}$ measured at 
pulling velocities $\mathbf{v}=\left\{v_{i}\right\}_{i=1}^{N}$. Since the $f_{i}$ are statistically independent, this probability reads:

$$
p(\mathbf{f} \mid \boldsymbol{\mu}, \mathbf{v})=\prod_{i=1}^{N} p_{1}\left(f_{i} \mid \boldsymbol{\mu}, v_{i}\right)
$$

The proceeding consists in simply maximizing (12) with respect to $\boldsymbol{\mu}$ [29, 30]; usually, this has to be done numerically. For any given $\mathbf{f}$ and $\mathbf{v}$ the corresponding set of parameters $\boldsymbol{\mu}^{*}=\boldsymbol{\mu}^{*}(\mathbf{f}, \mathbf{v})$ is called the maximum likelihood estimate.

Intuitively, the properties of this estimator are most easily understood within the framework of Bayesian inference [31, 32]. The quantity in (12) is called likelihood and plays a central role in the Bayesian approach. Extending the notion of "probability" in the sense of "degree of belief" to the model parameters $\boldsymbol{\mu}$, the joint probability $p(\mathbf{f}, \boldsymbol{\mu}, \mathbf{v})$ can be written in terms of conditional probabilities $p(\ldots \mid \ldots)$ either in the form $p(\boldsymbol{\mu} \mid \mathbf{f}, \mathbf{v}) p(\mathbf{f}, \mathbf{v})$ or in the form $p(\mathbf{f} \mid \boldsymbol{\mu}, \mathbf{v}) p(\boldsymbol{\mu}, \mathbf{v})$, yielding Bayes' theorem:

$$
p(\boldsymbol{\mu} \mid \mathbf{f}, \mathbf{v})=p(\mathbf{f} \mid \boldsymbol{\mu}, \mathbf{v}) p(\boldsymbol{\mu}, \mathbf{v})[p(\mathbf{f}, \mathbf{v})]^{-1} .
$$

The left hand side represents the "likeliness" of $\boldsymbol{\mu}$, given the data $\mathbf{f}, \mathbf{v}$, and hence is clearly of central interest for our purposes. Considering also the right hand side as a function of $\boldsymbol{\mu}$, it is equal to the likelihood from (12) times the so called prior probability $p(\boldsymbol{\mu}, \mathbf{v})$, encapsulating all our knowledge about $\boldsymbol{\mu}$ before the measurement, times a $\boldsymbol{\mu}$-independent factor. Thus, determining $p(\boldsymbol{\mu} \mid \mathbf{f}, \mathbf{v})$ by means of the right hand side of (13) provides the central "recipe of learning" within the Bayesian approach 31, 32].

Regarding actual practical application of Bayesian inference, the determination of the prior probability is the most problematic point. Different recipes for selecting an appropriate prior exist. Common choices are distributions which are uniform in the parameters or the logarithms of the parameters. Rigorous justifications are in general not possible and one is left with postulating some heuristic ad hoc Ansatz.

However, dynamic force spectroscopy usually provides large data sets, i.e. large $N$. Then the likelihood (12) develops a narrow peak in the region of its maximum $\boldsymbol{\mu}^{*}$ (see next section) and the prior $p(\boldsymbol{\mu}, \mathbf{v})$ in (13), though usually unknown in detail, can be considered as approximately constant, i.e. $p(\boldsymbol{\mu} \mid \mathbf{f}, \mathbf{v}) \propto p(\mathbf{f} \mid \boldsymbol{\mu}, \mathbf{v})$. Given f and $\mathbf{v}$, the likelihood (12) thus quantifies the "likeliness" that the "true" model parameters are $\boldsymbol{\mu}$.

The upshot of the above intuitive considerations is that maximizing (12) with respect to $\boldsymbol{\mu}$ should yield the best possible guess for the unknown true parameters. Furthermore, the statistical uncertainties of this estimate should be somehow related to the width of the likelihood. In the following subsection, we leave this intuitive level and turn to a more rigorous discussion of the asymptotic properties of the maximum likelihood estimator.

\section{B. Asymptotic Properties}

Let us assume that the rupture forces $f_{i}$ have been sampled according to the "true" distribution $p_{1}\left(f_{i} \mid \boldsymbol{\mu}_{0}, v_{i}\right)$ with unknown, "true" model parameters $\boldsymbol{\mu}_{0}$. For a given set of rupture forces $\mathbf{f}$ and pulling velocities $\mathbf{v}$ the maximum likelihood estimate can then be determined as described above. Upon repeating the entire set of $N$ pulling experiments with the same set of pulling velocities $\mathbf{v}$, a different set of rupture data $\mathbf{f}$ will be sampled, yielding a different maximum likelihood estimate $\boldsymbol{\mu}^{*}$. While the probability distribution of $\mathbf{f}$ is given by (12) with $\boldsymbol{\mu}=\boldsymbol{\mu}_{0}$, what can we say about the distribution of the maximum likelihood estimates $\boldsymbol{\mu}^{*}$ ?

To answer this question we first exploit the fact that in typical single-molecule pulling experiments for each pulling velocity several hundred rupture forces are measured. The resulting set of rupture force data $\mathbf{f}$ is thus quite large and it is convenient to rewrite the likelihood (12) as

$$
\begin{aligned}
p(\mathbf{f} \mid \boldsymbol{\mu}, \mathbf{v}) & =\exp \left\{-N s_{N}(\mathbf{f}, \boldsymbol{\mu}, \mathbf{v})\right\} \\
s_{N}(\mathbf{f}, \boldsymbol{\mu}, \mathbf{v}) & :=-N^{-1} \sum_{i=1}^{N} \ln p_{1}\left(f_{i} \mid \boldsymbol{\mu}, v_{i}\right) .
\end{aligned}
$$

Furthermore, we assume that rupture forces have been measured at $Z$ different pulling velocities $v_{\beta}, \beta=1, \ldots, Z$, and that the relative frequency with which the different pulling velocities $v$ are sampled, converges towards a well defined limit $\rho(v)=\sum_{\beta=1}^{Z} \rho_{\beta} \delta\left(v-v_{\beta}\right)$ for $N \rightarrow \infty$. Then it follows from the law of large numbers [33] that

$$
s_{N}(\mathbf{f}, \boldsymbol{\mu}, \mathbf{v}) \rightarrow s(\boldsymbol{\mu}):=-\left\langle\ln p_{1}(f \mid \boldsymbol{\mu}, v)\right\rangle_{1}
$$

for $N \rightarrow \infty$, where $\langle\cdots\rangle_{1}$ indicates an average over $f$ and $v$ with weight $p_{1}\left(f \mid \boldsymbol{\mu}_{0}, v\right) \rho(v)$. Hence, $s_{N}$ is an intensive, entropy-like quantity. Observing that $s(\boldsymbol{\mu})-s\left(\boldsymbol{\mu}_{0}\right)$ is a relative entropy of the form $\left\langle\ln \left(p_{1}\left(f \mid \boldsymbol{\mu}_{0}, v\right) / p_{1}(f \mid \boldsymbol{\mu}, v)\right\rangle_{1}\right.$, and using the fact that $p_{1}(f \mid \boldsymbol{\mu}, v)$ is normalized with respect to $f$ for every choice of the parameters $\boldsymbol{\mu}$, we obtain

$$
\begin{aligned}
& s(\boldsymbol{\mu})-s\left(\boldsymbol{\mu}_{0}\right)= \\
& \int d v \rho(v) \int d f p_{1}(f \mid \boldsymbol{\mu}, v)[R \ln R-R+1],
\end{aligned}
$$

with $R:=p\left(f \mid \boldsymbol{\mu}_{0}, v\right) / p(f \mid \boldsymbol{\mu}, v)$. Finally, using the inequality

$$
0 \leq \int_{1}^{R} d x \ln x=R \ln R-R+1 \quad \forall R,
$$

we see that $s(\boldsymbol{\mu})-s\left(\boldsymbol{\mu}_{0}\right) \geq 0$ and that $s(\boldsymbol{\mu})-s\left(\boldsymbol{\mu}_{0}\right)=0$ if the expression in the square brackets on the right hand side of (17) vanishes for all $f$. Thus, $s(\boldsymbol{\mu})$ has a unique absolute minimum at $\boldsymbol{\mu}=\boldsymbol{\mu}_{0}$ [33]. Since $s_{N}$ converges for large $N$ toward $s$ according to (16), also the minimum $\boldsymbol{\mu}^{*}$ of the former converges to the minimum $\boldsymbol{\mu}_{0}$ of the latter, i.e. the maximum likelihood estimate is a so-called consistent estimate [34]. 
For large, but finite $N$ values, $\boldsymbol{\mu}$ will be close to $\boldsymbol{\mu}^{*}$. Consequently, we can expand $s_{N}(\mathbf{f}, \boldsymbol{\mu}, \mathbf{v})$ up to second order about its minimum at $\boldsymbol{\mu}^{*}$ and neglecting terms of order $\mathcal{O}(1 / \sqrt{N})$, the Hessian matrix of $s_{N}\left(\mathbf{f}, \boldsymbol{\mu}^{*}, \mathbf{v}\right)$ can be replaced by the Hessian $H=H\left(\boldsymbol{\mu}_{0}\right)$ of $s\left(\boldsymbol{\mu}_{0}\right)$, which is generically positive definite, i.e.

$$
s_{N}\left(\mathbf{f}, \boldsymbol{\mu}^{*}+\boldsymbol{\Delta}, \mathbf{v}\right)=s_{N}\left(\mathbf{f}, \boldsymbol{\mu}^{*}, \mathbf{v}\right)+\boldsymbol{\Delta}^{\dagger} H \boldsymbol{\Delta} / 2 .
$$

For large $N$ this is a very good approximation for all $\boldsymbol{\mu}$ values, and $p(\mathbf{f} \mid \boldsymbol{\mu}, \mathbf{v})$ approaches a very sharply peaked Gaussian about $\boldsymbol{\mu}^{*}$,

$$
p(\mathbf{f} \mid \boldsymbol{\mu}, \mathbf{v}) \propto \exp \left\{-N\left(\boldsymbol{\mu}-\boldsymbol{\mu}^{*}\right)^{\dagger} H\left(\boldsymbol{\mu}-\boldsymbol{\mu}^{*}\right) / 2\right\} .
$$

We can now determine the first moments of the distribution of the maximum likelihood estimate $\boldsymbol{\mu}^{*}$ (upon many repetitions of the same experiment). Differentiating (19) and choosing $\boldsymbol{\Delta}=\boldsymbol{\mu}_{0}-\boldsymbol{\mu}^{*}$, results in

$$
\boldsymbol{\mu}^{*}-\boldsymbol{\mu}_{0}=-H^{-1} \partial s_{N}\left(\mathbf{f}, \boldsymbol{\mu}_{0}, \mathbf{v}\right) / \partial \boldsymbol{\mu} .
$$

Averaging over $\mathbf{f}$ yields zero in the right hand side, as can be inferred from (15), (16) and the fact that $\boldsymbol{\mu}_{0}$ is the minimum of $s$. Hence,

$$
\left\langle\boldsymbol{\mu}^{*}\right\rangle=\boldsymbol{\mu}_{0},
$$

where $\langle\cdots\rangle$ indicates an average over $\mathbf{f}$ with weight $p\left(\mathbf{f} \mid \boldsymbol{\mu}_{\mathbf{0}}, \mathbf{v}\right)$ for a given set of pulling velocities $\mathbf{v}$. Equation (22) thus shows that the maximum likelihood estimate is "unbiased" for large $N$.

With (21) the determination of the second moments is straightforward. Using

$$
\left\langle\frac{\partial}{\partial \mu_{i}} s_{N}\left(\mathbf{f}, \boldsymbol{\mu}_{0}, \mathbf{v}\right) \frac{\partial}{\partial \mu_{j}} s_{N}\left(\mathbf{f}, \boldsymbol{\mu}_{0}, \mathbf{v}\right)\right\rangle=\frac{1}{N} H_{i j},
$$

gives the covariance matrix for the maximum likelihood estimate:

$$
\left\langle\left[\boldsymbol{\mu}^{*}-\boldsymbol{\mu}_{0}\right]\left[\boldsymbol{\mu}^{*}-\boldsymbol{\mu}_{0}\right]^{\dagger}\right\rangle=(N H)^{-1} .
$$

Observing that $\left(\mathrm{NH}^{-1}\right.$ is also the covariance matrix of the distribution from (20) we arrive at our

First main conclusion: For any given, sufficiently large data set $\mathbf{f}$, the expected deviation of the concomitant maximum likelihood estimate $\boldsymbol{\mu}^{*}$ from the "true" parameters $\boldsymbol{\mu}_{0}$ immediately follows from the "peak-width" of likelihood (12), considered as a function of $\boldsymbol{\mu}$.

Similarly, using the central limit theorem, one can show (see Appendix $\mathrm{A}$ ), that $\boldsymbol{\mu}^{*}$ is Gaussian distributed, yielding with (20) our

Second main conclusion: Apart from the peak position and a normalization factor, the likelihood (12) for one given data set $\mathbf{f}$ looks practically the same as the distribution of the maximum likelihood estimates $\boldsymbol{\mu}^{*}$ from many repetitions of the $N$ pulling experiments.

\section{Cramér-Rao bound}

It should be noted that, in order to derive the above two main conclusions, we did not make any use of the Bayesian formalism (13) at all. The latter only served to acquire an intuitive idea about the meaning of the likelihood (12). At this intuitive level, we have seen that the left hand side of (13) is very well approximated by the sharply peaked Gaussian in (20) and hence it is reasonable to expect that its maximum $\boldsymbol{\mu}^{*}$ should be the best possible guess for the unknown true parameters $\boldsymbol{\mu}_{0}$ that possibly can be inferred from a given set of data $f$. A more rigorous line of reasoning starts with an arbitrary "recipe" $\tilde{\boldsymbol{\mu}}(\mathbf{f})$ of estimating the true parameters $\boldsymbol{\mu}_{0}$ from a given data set $\mathbf{f}$. The only assumption is that this recipe is unbiased, i.e. upon repeating the same experiment many times, the "true" parameters are recovered on average, $\langle\tilde{\boldsymbol{\mu}}(\mathbf{f})\rangle=\boldsymbol{\mu}_{0}$. By generalizing the well-known Cramér-Rao inequality [33], which in turn is basically a descendant of the Cauchy-Schwarz inequality, one can show [34] for any such "recipe" $\tilde{\boldsymbol{\mu}}(\mathbf{f})$ that

$$
\left\langle\left[\tilde{\boldsymbol{\mu}}-\boldsymbol{\mu}_{0}\right]\left[\tilde{\boldsymbol{\mu}}-\boldsymbol{\mu}_{0}\right]^{\dagger}\right\rangle-(N H)^{-1} \geq 0,
$$

i.e. the matrix in the left hand side is non-negative definite. Comparison with (24) yields our

Third main conclusion: There is no unbiased estimator $\tilde{\boldsymbol{\mu}}$ of the true parameters $\boldsymbol{\mu}_{0}$ which on the average outperforms the maximum likelihood estimate $\boldsymbol{\mu}^{*}$.

The remaining possibility that a biased estimator may be even better is rather subtle to treat rigorously, but intuitively this seems quite unlikely. Furthermore, in the above conclusion we exploited the relation (24) which is strictly correct only for asymptotically large $N$. Finally, also the criterion of minimizing the left hand side in (25) itself is in principle debatable, but hardly in practice.

\section{Parameter Inference: Main steps for the practical application}

We now briefly summarize the main steps of the maximum likelihood method for evaluating single-molecule pulling experiments. The first step consists in specifying the dependence of the rupture force probability $p_{1}(f \mid \boldsymbol{\mu}, v)$ on the model parameters $\boldsymbol{\mu}$ and the pulling velocity $v$ within any given theoretical description. Then for the set of rupture forces $\mathbf{f}=\left\{f_{i}\right\}_{i=1}^{N}$ and corresponding pulling velocities $\mathbf{v}=\left\{v_{i}\right\}_{i=1}^{N}$ the logarithm of the likelihood (14)

$$
-N s_{N}(\mathbf{f}, \boldsymbol{\mu}, \mathbf{v})=\sum_{i=1}^{N} \ln p_{1}\left(f_{i} \mid \boldsymbol{\mu}, v_{i}\right)
$$

is maximized with respect to the model parameters $\boldsymbol{\mu}$. Usually this step has do be accomplished numerically. The position of the maximum defines the most probable parameters $\boldsymbol{\mu}^{*}$ which are on average closer to the true 
model parameters than any other estimate. The statistical uncertainties of the parameters can then be estimated as

$$
\left\langle\left[\boldsymbol{\mu}^{*}-\boldsymbol{\mu}_{0}\right]\left[\boldsymbol{\mu}^{*}-\boldsymbol{\mu}_{0}\right]^{\dagger}\right\rangle \approx\left(N H_{N}\right)^{-1},
$$

where $H_{N}$ denotes the Hessian matrix of $s_{N}$ evaluated at the most probable parameters $\boldsymbol{\mu}^{*}$ and $\boldsymbol{\mu}_{0}$ the true model parameters. Moreover, the distribution of the maximum likelihood estimate is Gaussian with mean $\boldsymbol{\mu}_{0}$.

\section{APPLICATION TO SINGLE-MOLECULE FORCE SPECTROSCOPY: BELL'S MODEL}

Combining the result (5) with approximations (7), (9) [or (10), (11)], one can apply the maximum likelihood approach from the previous section to deduce the rate parameters for any exponentially increasing escape rate (11). In what follows, however, we will focus on two efficient rate approximations [see Eqs. (28) and (35) below], which allow one to evaluate the integral from Eq. (6) analytically.

The first common approximation, originally due to Bell [11], consist in the linearization of the force dependent potential barrier according to (3) and in neglecting the force dependence of the pre-exponential factor $\omega(f)$ in the Kramers rate (1), resulting in [cf. (3) and Fig. 2]

$$
k(f)=k_{0} \exp \left(\frac{x_{\mathrm{b}} f}{k_{\mathrm{B}} T}\right)=: \exp (\lambda+\alpha f),
$$

where $k_{0}:=\omega(0) \exp \left(-E_{\mathrm{b}}(0) / k_{\mathrm{B}} T\right)$ is the force-free dissociation rate, $x_{\mathrm{b}}$ the dissociation length (distance between potential well and barrier), and $\lambda:=\ln k_{0}, \alpha:=$ $x_{\mathrm{b}} / k_{\mathrm{B}} T$ are convenient abbreviations.

Substituting Eq. (28) into (5), (6) and going over to $f$ as independent variable, a straightforward calculation yields the probability density of rupture events for Bell's model:

$$
p_{1}(f \mid \boldsymbol{\mu}, v)=\frac{e^{\lambda+\alpha f}}{\kappa v} \exp \left(-\frac{e^{\lambda}}{\kappa v} \frac{e^{\alpha f}-1}{\alpha}\right) .
$$

The rupture force density (29) is conditioned on $\boldsymbol{\mu}=$ $(\lambda, \alpha)$, and $v$. As usual, we assume that the pulling velocity $v$ is known exactly for each measurement, and similarly for the elasticity $\kappa$ appearing on the right hand side of (29). The remaining model parameters to be estimated from a given set of rupture forces $\mathbf{f}=\left\{f_{i}\right\}_{i=1}^{N}$ measured at pulling velocities $\left\{v_{\beta}\right\}_{\beta=1}^{Z}$ with relative frequencies $\rho_{\beta}$ are therefore $\boldsymbol{\mu}=(\lambda, \alpha)$.

\section{A. Statistical uncertainties of Bell's model}

For the above specified model we can calculate $s(\boldsymbol{\mu})=$ $-\left\langle\ln p_{1}(f \mid \boldsymbol{\mu}, v)\right\rangle_{1}$ as defined in (16) analytically, if the dimensionless quantity $\tau_{\beta}:=e^{\lambda_{0}} /\left(\kappa v_{\beta} \alpha_{0}\right)$ is small for all pulling velocities. In fact, $\tau_{\beta}<1$ is equivalent to the assumption that the distribution of rupture events $p_{1}\left(f \mid \boldsymbol{\mu}_{0}, v_{\beta}\right)$ has a maximum at some force $f_{\beta}^{*}>0$. Details of the calculations are given in Appendix B, resulting in:

$$
\begin{aligned}
s(\boldsymbol{\mu}) & =-\lambda+\sum_{\beta=1}^{Z} \rho_{\beta} s_{\beta}(\boldsymbol{\mu})+\mathcal{O}\left(\tau_{\beta}\right), \\
s_{\beta}(\boldsymbol{\mu}) & :=\ln \left(\kappa v_{\beta}\right)+\eta\left(\mathcal{C}+\ln \tau_{\beta}\right)+\frac{e^{\lambda} \Gamma(\eta+1)}{\kappa v_{\beta} \alpha\left[\tau_{\beta}\right]^{\eta}}
\end{aligned}
$$

with $\eta:=\alpha / \alpha_{0}, \mathcal{C} \approx 0.577$ Euler's constant, and $\Gamma(\cdot)$ the Gamma function. Differentiating (30) twice with respect to the model parameters, a straightforward calculation yields the Hessian $H=H\left(\boldsymbol{\mu}_{0}\right)$ of $s\left(\boldsymbol{\mu}_{0}\right)$. Finally, inverting this $(2 \times 2)$ matrix, we obtain the variance of the maximum likelihood estimate of the two parameters:

$$
\begin{aligned}
\left\langle\left(\alpha^{*}-\alpha_{0}\right)^{2}\right\rangle & \approx \frac{\alpha_{0}^{2}}{N} \frac{1}{\frac{\pi^{2}}{6}+\sigma^{2}(\ln (r))}, \\
\left\langle\left(\lambda^{*}-\lambda_{0}\right)^{2}\right\rangle & \approx \frac{1}{N} \frac{\sum_{\beta=1}^{Z} \frac{N_{\beta}}{N}\left(\alpha_{0}^{2}\left\langle f_{\beta}\right\rangle^{2}+\frac{\pi^{2}}{6}\right)}{\frac{\pi^{2}}{6}+\sigma^{2}(\ln (r))},
\end{aligned}
$$

where

$$
\sigma^{2}(\ln (r))=\sum_{\beta=1}^{Z} \rho_{\beta} \ln ^{2}\left(r_{\beta}\right)-\left(\sum_{\beta=1}^{Z} \rho_{\beta} \ln \left(r_{\beta}\right)\right)^{2}
$$

is the variance of the logarithm of the loading rate $r:=\kappa v$ and

$$
\left\langle f_{\beta}\right\rangle=-1 / \alpha_{0}\left(\mathcal{C}+\ln \left(e^{\lambda_{0}} /\left(r_{\beta} \alpha_{0}\right)\right)\right)
$$

the expected rupture force at loading rate $r_{\beta}$ (again neglecting terms of order $\mathcal{O}\left(\tau_{\beta}\right)$ ). For practical application of Eqs. (31) and (32) the true model parameters $\boldsymbol{\mu}_{0}$ in the right-hand sides of the equations have to be replaced by the concomitant maximum likelihood estimate $\boldsymbol{\mu}^{*}$. For large $N$ this is a very good approximation.

In single-molecule pulling experiments only a limited range of pulling velocities $v$ is accessible, i.e. $v_{\beta} \in$ $\left[v_{\min }, v_{\max }\right]$. Now the question arises: for which distribution of pulling velocities $\rho(v)$ do the statistical uncertainties (31), (32) of the estimated parameters become minimal? Recognizing that the variance of the maximum likelihood estimate of $\alpha$ depends on the distribution of the pulling velocities solely via the term $\sigma^{2}(\ln (r))$ in the denominator of (31), while this distribution enters into the expression for the statistical uncertainties of $\lambda^{*}$ also via the terms $\left\langle f_{\beta}\right\rangle$ in the numerator of (32), we see, that it is, in general, not possible to simultaneously minimize the two uncertainties. Given a fixed number $N$ of pulling experiments, the minimization of the variance $\left\langle\left(\alpha^{*}-\alpha_{0}\right)^{2}\right\rangle$ of $\alpha^{*}$ is equivalent to a maximization of $\sigma^{2}(\ln (r))$. Under the constraint $v_{\beta} \in\left[v_{\min }, v_{\max }\right]$ for all 
pulling velocities $v_{\beta}$, this maximum is obviously reached if half of the rupture forces have been sampled at a pulling velocity as large as possible, i.e. $v_{\max }$, and the other half at a pulling velocity as small as possible, i.e. $v_{\min }$. This result is independent of the values of the true model parameters $\boldsymbol{\mu}_{0}$. Regarding the distribution of $\lambda^{*}$, the situation is more complicated. Given the analytic expression (32) for the error, the calculation is straightforward, but not shown here for the following two reasons. The "best" choice consists again in sampling just at the two extreme pulling velocities $v_{\min }$ and $v_{\max }$. The relative number of pulling experiments for each of the two pulling velocities is, however, non-trivial and depends on the values of the true model parameters $\boldsymbol{\mu}_{0}$. It is, therefore (in contrast to the result for $\alpha^{*}$ ), only of limited use for a real experiment.

\section{B. Illustration for computer generated data}

We now illustrate the findings from sections $\amalg I \mathrm{~B}$ and IIIC To avoid uncontrollable experimental inaccuracies and uncertainties regarding the "true" model and the "true" model parameters $\boldsymbol{\mu}_{0}$, we numerically generated synthetic rupture data $\mathbf{f}$ by "simulating" an idealized experiment on the computer according to the probabilistic "laws" (28), (29) with given parameters $\boldsymbol{\mu}=\boldsymbol{\mu}_{0}$. Hence all remaining uncertainties are statistical finite $N$ effects. Fig. 3 shows the results for 10000 repetitions of a computer experiment, each sampling $N=400$ rupture forces f according to (29) with experimentally realistic parameter values $\alpha_{0}=0.1 \mathrm{pN}^{-1}$ and $\lambda_{0}=-5$. Since twodimensional distributions are difficult to compare graphically, we focus on the marginal distributions. For each of the 10000 experiments, the maximum $\boldsymbol{\mu}^{*}=\left(\lambda^{*}, \alpha^{*}\right)$ of the likelihood (12), considered as a function of $\boldsymbol{\mu}$, was determined numerically. The distribution of the resulting $\lambda^{*}$ - and $\alpha^{*}$-values are depicted as histograms in Fig. 3. The standard deviations of the maximum likelihood estimate for $\lambda$ and $\alpha$, determined from the 10000 experiments are $s\left(\lambda^{*}\right)=0.20$ and $s\left(\alpha^{*}\right)=0.0023 \mathrm{pN}^{-1}$ respectively. These values coincide with those obtained from the analytical approximations (31)-(34) up to the third non-vanishing digit. Replacing the parameters $\boldsymbol{\mu}_{0}$ on the right-hand side of (31), (32) by the maximum likelihood estimate $\boldsymbol{\mu}^{*}$ for one given data set thus provides reliable estimates for the statistical uncertainties.

Furthermore, for the first 15 of the 10000 experiments, after integrating over the other parameter in the likelihood (12), shifting the peak position from $\mu_{i}^{*}$ to $\mu_{i, 0}$, and normalizing, the resulting marginal distributions were plotted in Fig. 3. They closely agree with the histograms. These observations illustrate very convincingly our two conclusions from section III B above. In particular, finite$N$ corrections are apparently very small for the typical parameter values used in this example. Also the practically perfect Gaussianity of the distributions is as expected, cf. (20). (a)

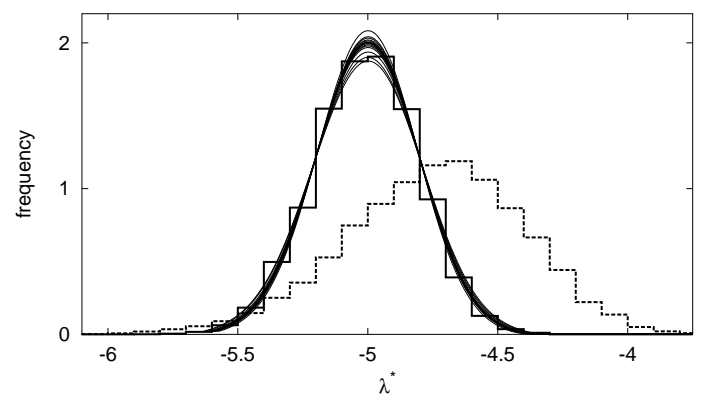

(b)

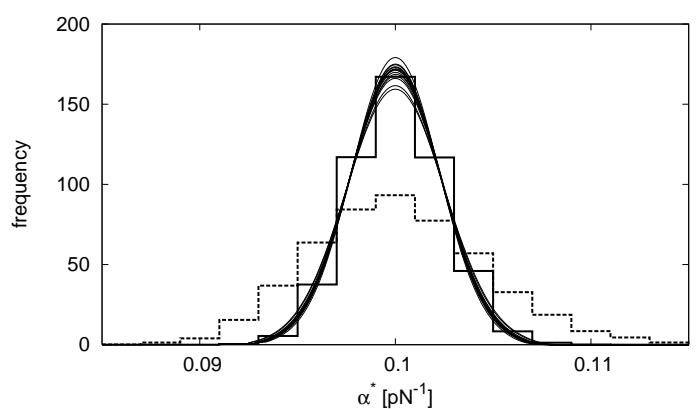

FIG. 3: Solid histogram: Distributions of the first and second components of the maxima $\boldsymbol{\mu}^{*}=\left(\lambda^{*}, \alpha^{*}\right)$ of the likelihood (12) for 10000 "computer experiments". For each of them, $N=400$ rupture forces $f$ were sampled according to (29), 100 for each of the 4 loading rates $\kappa v=50,200,1000,5000$ $\mathrm{pN} / \mathrm{s}$ and with "true" parameters $\lambda_{0}=-5$ and $\alpha_{0}=0.1$ $\mathrm{pN}^{-1}$. These are typical numbers in "real experiments" [1]. For sake of better visibility the bin-width of the histograms is much larger than the optimal bin width for a Gaussian distribution (Appendix C). Thin lines: Likelihood (12) for the first 15 of the 10000 experiments after integrating over the other component, shifting the maximum to $\mu_{i, 0}$, and normalizing (some are almost indistinguishable). Dotted histogram: Distribution of the estimates for $\lambda$ and $\alpha$ according to the "standard method", as described in the main text.

Let us finally compare the performance of the maximum likelihood estimate with that of the most widely used "recipe" of parameter estimation in the field of single-molecule pulling experiments. This consist of the following steps: (i) Fit a Gaussian to the observed rupture force distribution for a fixed pulling velocity $v$ and approximate the most probable rupture force $f^{*}$ by the maximum of that Gaussian. (ii) Plot $f^{*}$ for different $v$ versus $\ln (v)$ and fit the resulting points by a straight line. (iii) Assume that the model (28), (29) is applicable and deduce its model parameters $\boldsymbol{\mu}=(\lambda, \alpha)$ from the slope and the axis intercept of the straight line as detailed e.g. in [1, 12, 15, 16, 35]. We have applied this procedure to each of the 10000 experiments in Fig. 3 and plotted the distribution of the resulting estimates for $\lambda$ and $\alpha$ in Fig. 3. The systematic bias of the estimate for $\lambda$ can be traced back to fitting a Gaussian, which is symmetric about its maximum, to an asymmetric "true" distribution (29) [35], while the suboptimal variance of 
the estimate for both $\lambda$ and $\alpha$ signals that quite some information is lost by only considering the most probable rupture forces $f^{*}$. Hence, the maximum likelihood estimate represents a substantial improvement compared to the so far "standard method" of data evaluation in this field. This is in agreement with our conclusion from section 【IC. We have also directly compared the maximum likelihood estimate with other known "recipes" of evaluating single-molecule rupture data, e.g. [35]. In all cases we found that the maximum likelihood was superior.

\section{EXTENSION OF BELL'S MODEL}

\section{A. Rate Ansatz}

As can be seen from Fig. 2, a linearization (28) of the force dependent potential barrier $E_{\mathrm{b}}(f)$ is relatively good for small forces. For a larger forces, the distance between potential extrema decreases, leading to a weaker sensitivity of the barrier height to force variations upon further pulling than in Eq. (28).

Models including this effect, in general, rely on some assumptions concerning the shape of the energy landscape. Typical choices are Morse potentials, harmonic potentials with a cusp barrier, two parabolas at the potential extrema joined at a midpoint, and linear-cubic potentials [6, 22, 23, 24, 25, 26, 26, 27]. It has been suggested in 25] that for sufficiently high barriers, i.e. forces substantially smaller than the critical force, the dissociation rate can be written in a unique form

$$
k(f)=(1-\gamma \alpha f / \epsilon)^{1 / \gamma-1} e^{\lambda+\epsilon\left[1-(1-\gamma \alpha f / \epsilon)^{1 / \gamma}\right]}
$$

with three model parameters $\boldsymbol{\mu}=(\lambda, \alpha, \epsilon)$ and fixed exponent $1 / \gamma$. Here, $\lambda$ and $\alpha$ have the same physical meaning as in Eq. (28), and $\epsilon:=E_{\mathrm{b}}(0) / k_{\mathrm{B}} T$ stands for the force-free activation energy barrier in units of the thermal energy $k_{\mathrm{B}} T$.

The extra parameter $\gamma$ controls the manner in which the barrier height decreases with the applied force. We note that, physically, this parameter should be in the range $\gamma \in(0,1]$ since $\gamma \leq 0$ would imply a positive barrier for all $f>0$. On the other hand, the first derivative of the barrier height equals minus the distance between the potential extrema corresponding to a given force value. Since we expect this distance to decrease with the force, we conclude that the second force derivative of the barrier height must be positive, excluding $\gamma$-values greater than 1. Specifically, for $\gamma=1$ the parameter $\epsilon$ drops out and one recovers Bell's model (28), $\gamma=2 / 3$ reproduces the Kramers rate for a cubic reaction potential, and $\gamma=$ $1 / 2$ corresponds to a parabolic potential well with a cusp barrier.

Substituting Eqs. (4), (35) into (2), one derives the survival probability of the bond up to force $f$ :

$$
n(f)=\exp \left(-\frac{e^{\lambda}}{\kappa v} \frac{e^{\epsilon\left[1-(1-\gamma \alpha f / \epsilon)^{1 / \gamma}\right]}-1}{\alpha}\right),
$$

and the probability density of rupture events follows from Eq. (5).

As already mentioned, the application of Kramers reaction rate theory requires that the potential barrier $E_{\mathrm{b}}(f)$ be sufficiently high (compared to the thermal energy $\left.k_{\mathrm{B}} T\right)$. Thus, all approximations are only valid for forces substantially smaller than the critical force at which the barrier vanishes, $f_{\mathrm{c}}=\epsilon /(\gamma \alpha)$.

The above discussion suggests that dynamic force spectroscopy should, in principle, provide the possibility, not only to determine the force-free dissociation rate $k_{0}=\exp (\lambda)$ and the dissociation length $x_{\mathrm{b}}$, but also the force-free activation energy barrier $E_{\mathrm{b}}(0)=\epsilon k_{\mathrm{B}} T$. Naturally, the question arises how accurate these estimates will be and whether the inferred values critically depend on the chosen theoretical model, in particular on the value of the parameter $\gamma$. These questions will be addressed next.

\section{B. Numerical experiment}

There is an ongoing debate in literature about which of the three exponents, i.e. $\gamma=1, \gamma=2 / 3$, or $\gamma=1 / 2$, is most appropriate to use when evaluating experimental rupture data 22, 23, 24, 25, 26]. Taking for granted that one of the three models approximates the "truth" satisfactorily, choosing $\boldsymbol{\mu}=\boldsymbol{\mu}^{*}$ is - according to our above conclusions - the closest one can get to the "full truth" on the basis of one given data set $\mathbf{f}$. In case of disagreement about the "true" $\gamma$-value, a fully objective selection criterion seems unavailable in principle. In practice, the usual criterion is the comparison with the basic "true" quantity observed experimentally, namely the distribution of rupture forces.

For the example shown in figure 4 we sampled $N=$ 2000 rupture forces $\mathbf{f}$ according to the distribution (5) with rate (35). In the simulations, we have taken $\gamma=2 / 3$ and realistic parameter values $\lambda_{0}=-5, \alpha_{0}=0.1 \mathrm{pN}^{-1}$, $\epsilon_{0}=15$.

In order to test the maximum likelihood method, for the resulting data set we determined the estimate $\boldsymbol{\mu}^{*}$ for several possible $\gamma$-values. Since in a real experiment, the value of the exponent $\gamma$ is not known a priori, also during the fitting, the $\gamma$-values used were not necessarily coincident with the "true" value used for data generation. The bin-width of the histograms in Fig. 4 was chosen as $h_{\beta}=2.83 s_{N_{\beta}} N_{\beta}^{-1 / 3}$, where $s_{N_{\beta}}$ is the standard deviation of the rupture forces measured at pulling velocity $v_{\beta}$. As discussed in Appendix $\mathrm{C}$, this is the optimal choice of the bin-width for the Bell model; although this model is in fact not the true one in our numerical experiment, this choice of the bin-width remains suitable, as every distribution separately can be very well fitted with Eq. (29).

Comparing in Fig. 4 the resulting distributions $p_{1}\left(f \mid \boldsymbol{\mu}^{*}, v\right)$ for three different $\gamma$ values, we observe the following. Already the two-parametric Bell's Ansatz $(\gamma=1)$ reproduces the experimental distribution of rup- 


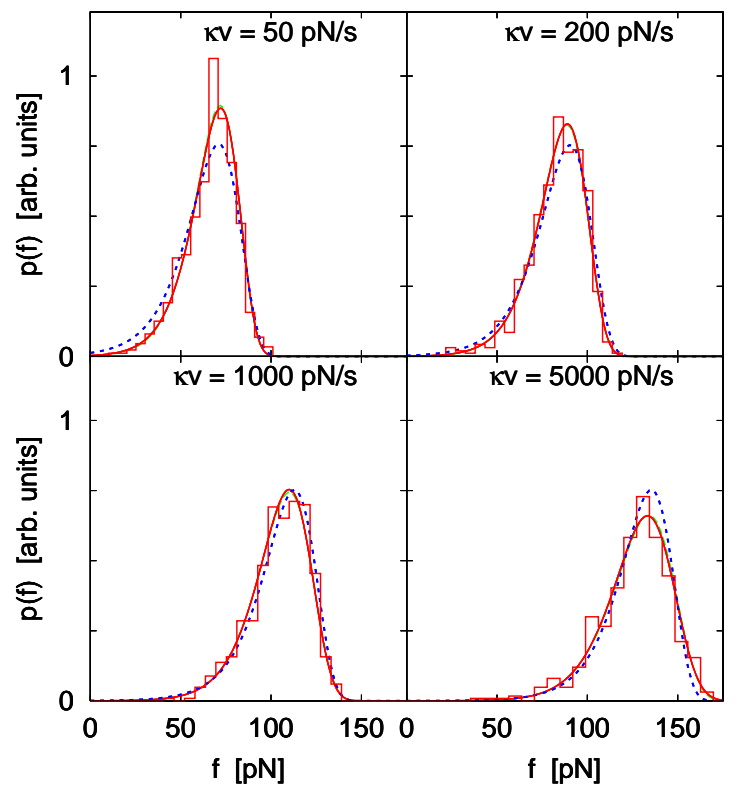

FIG. 4: (Color online) Rupture force distribution for different loading rates $\kappa v$. Histograms: numerically generated rupture forces according to (5), (35) with $\gamma=2 / 3, \lambda_{0}=-5, \alpha_{0}=0.1$ $\mathrm{pN}^{-1}, \epsilon_{0}=15$. For each $\kappa v$, we sampled 500 forces, i.e. $N=$ 2000. The bin-width is chosen according to eq. (C6). The maximum likelihood fits $p_{1}\left(f \mid \boldsymbol{\mu}^{*}, v\right)$ according to (5), (35) for $\gamma=1 / 2$ and $\gamma=2 / 3$ (red solid) are not distinguishable within the line width in this plot. For $\gamma=1 / 2$, the fit parameters have the following values: $\lambda=-5.37, \alpha=0.110$, and $\epsilon=$ 17.2. For $\gamma=2 / 3$, the fit results are slightly closer to the true parameter values, namely, $\lambda=-5.20, \alpha=0.104$, and $\epsilon=$ 14.64. Blue dashed distribution: same for Bell's Ansatz $\gamma=1$, with fit results $\lambda=-3.81$ and $\alpha=0.072$. Upon repeating the entire "numerical experiment", the resulting plots always look practically the same.

ture forces with reasonable accuracy, see the dotted line in Fig. 4. However, if one increases the number of fit parameters to three, one obtains the fit distributions notably different from the Bell's curves, see the solid lines corresponding to $\gamma=1 / 2$ and $\gamma=2 / 3$. We also note that the difference between the curves corresponding to these two values of $\gamma$ is smaller than the line thickness. This means that if one treats the exponent $\gamma$ as a fourth fit parameter, then its precise value cannot be determined by fitting the experimental rupture force distribution. With respect to the three remaining fit parameters, their values are rather close to each other for the fits with $\gamma=1 / 2$ and $\gamma=2 / 3$, with the largest discrepancy between the fit values of the force-free barrier height (see the caption in Fig. (4).

When fitting the real experimental data, one does not know a priori the true value of the exponent $\gamma$. It is therefore of interest to find out, how the remaining fit parameter values depend on the assumption with respect to this quantity. Fig. 5 shows the fit results obtained for different assumed values of $\gamma$ within the physically meaningful range. All of the fitting curves obtained for different $\gamma$-values from Fig. 5 coincided within the line thickness. We observe that the resulting fit values of the force-free escape rate $e^{\lambda}$ and the dissociation length $k_{\mathrm{B}} T \alpha$ are not very sensitive to the choice of the exponent $\gamma$. At the same time, the force-free barrier height value inferred from the fit depends approximately linearly on the choice of this parameter, and can assume values differing by as much as a factor of 2 at extreme $\gamma$-values. This means that when fitting the experimental data, the value of the force-free barrier height will be determined with the least accuracy.

In view of these observations, an interesting question arises: are the approximations (24) and (31)-(34) for the statistical uncertainties of the model parameters still valid for those models, about which we (in our case) know that they are not true? To study this point, we have repeated the above described procedure for 10000 data sets, each generated in the same way and with same "true" parameters as the data set shown in Fig. 4. The distributions of the inferred parameters $\boldsymbol{\mu}^{*}$ for the three different $\gamma$-values are depicted in Fig. 6 as histograms. Following section IIIB they should be bell-shaped with variance given by Eq. (24). We have evaluated this expression at the mean values of the inferred parameters $\boldsymbol{\mu}^{*}$ for each of the three $\gamma$-values respectively. For $\gamma=2 / 3$ and $\gamma=1 / 2$ this had to be done numerically, whereas for $\gamma=1$, Eqs. (31)-(34) could be employed. The resulting distributions, centered about the mean value of the inferred parameters, are shown in Fig. 6 as solid lines. For $\lambda$ and $\alpha$ they closely agree to the histograms. With respect to the parameter $\epsilon$, the approximated variance agrees very well with the empirically determined, but finite- $N$ corrections to the full distributions which are not symmetric about their center are apparent.

In conclusion, although the models for $\gamma=1$ and $\gamma=1 / 2$ are not the true models, equation (24) still yields very good approximations for the statistical uncertainties of the parameters, i.e. for their distribution upon repeating the same experiment many times. Nevertheless, comparing these distributions to the distribution of the parameters for the "true" gamma-value $\gamma=2 / 3$, we see, that by choosing the wrong model, the systematic deviations to the parameters are much larger than the statistical uncertainties. As one will, in general, be unsure about the true underlying energy landscape, and thus about the "true" $\gamma$-value, this point is essential if one wishes to use the inferred parameter values in another context than the interpretation of single-molecule pulling experiments.

We would like to mention that the deviations between the distributions resulting from the different $\gamma$ values increase with the pulling velocity [25]. Hence, by increasing the range of accessible pulling velocities $\left[v_{\min }, v_{\max }\right]$ a clearer distinction between the models is possible. However, for precise measurements with the AFM the loading rate $\kappa v$ is limited to a few orders of magnitude, comparable with our values. 

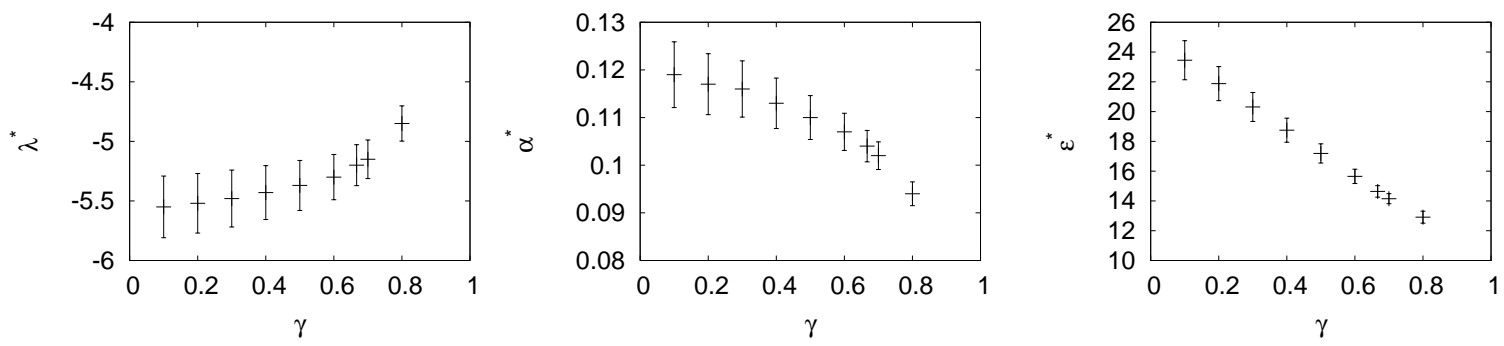

FIG. 5: Maximum likelihood fit values for the data set from Fig. 4 fitted with rupture force distribution (5) supplemented with the escape rate (36). Each data point was obtained by fitting the same data set, but assuming a different value of the parameter $\gamma$.
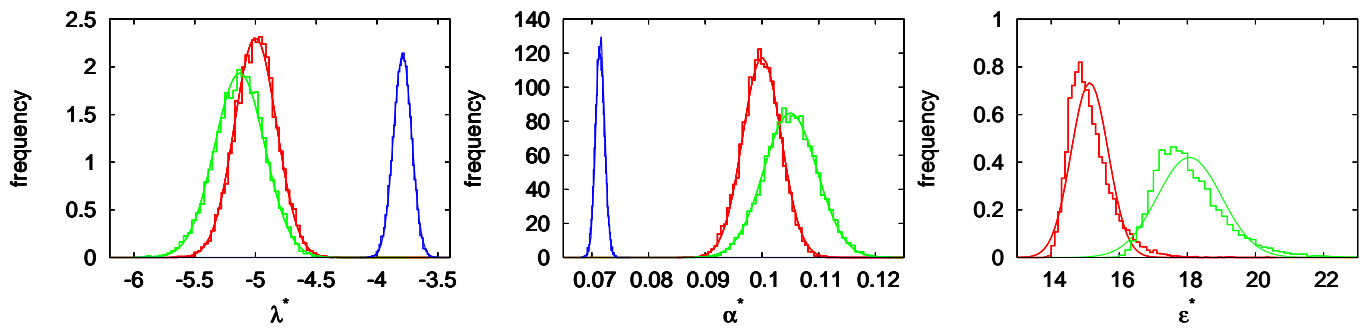

FIG. 6: (Color online) Histograms: Numerically determined distribution of the single components of the maxima $\boldsymbol{\mu}^{*}=$ $\left(\lambda^{*}, \alpha^{*}, \epsilon^{*}\right)$ of the likelihood (12), (35) with $\gamma=1 / 2$ (green), $\gamma=2 / 3$ (red) and $\gamma=1$ (blue) for 10000 "computer experiments". For all data sets the rupture forces were generated numerically according to (5), (35) with $\gamma=2 / 3, \lambda_{0}=-5, \alpha_{0}=0.1 \mathrm{pN}^{-1}$, $\epsilon_{0}=15$. For each $\kappa v$, we sampled 500 forces, i.e. $N=2000$. The bin-width of the histograms is $h_{\beta}=3.49 s_{N_{\beta}} N_{\beta}^{-1 / 3}(\operatorname{see}$ Appendix (C). Solid lines: Gaussian approximations to the distributions with covariance matrices (24). They have been shifted so that their maximum coincides with the mean of the empirically determined distributions. For sake of better visibility the distributions for $\gamma=1$ have been rescaled by an appropriate value.

\section{CONCLUSIONS}

In this work we have shown that the maximum likelihood approach is an extremely simple, general, and powerful method for parameter estimation in the contect of single-molecule force spectroscopy. For large data sets it outperforms all other estimates. Furthermore, approximations to the statistical uncertainties of the parameters are available once the parameters are estimated. In the case of the standard Bell model we were able to derive an analytical expression for these uncertainties in terms of the model parameters and the distribution of the applied loading rates. For more general models, the uncertainties can be determined numerically.

When fitting the experimental data, one usually adopts some functional form of the force-dependent escape rate involving several fit parameters. By means of a numerical example, we have demonstrated that the largest number of such parameters that can be determined from the experiment is three. These parameters are related to the force-free value of the rate, the dissociation length, and the barrier height in the absence of the force. Furthermore, when fitting the experimental rupture force dis- tributions, one needs to make an additional assumption about the manner in which the escape rate decreases with the applied force. While the fit values of the force-free escape rate and the dissociation length depend only weakly on this assumption, the value of the force-free barrier height can be determined much less reliably. We have shown that even if the model adopted for the description of the experiment is not the true one but predicts distributions of rupture forces similar to the measured distribution, the statistical uncertainties found from the maximum likelihood method very well approximate the dispersion of the estimated parameters upon repeating the same experiment many times. Often these uncertainties are much smaller than the systematic error resulting from choosing the "wrong" model.

\section{Acknowledgments}

We are grateful to the Deutsche Forschungsgemeinschaft (RE 1344/3-1, and SFB 613) for financial support of this work. 


\section{APPENDIX A: ASYMPTOTIC DISTRIBUTION OF THE MAXIMUM LIKELIHOOD ESTIMATE}

In this section we proof the second main conclusion from section IIIB, namely that the distribution of the maximum likelihood estimate $\boldsymbol{\mu}^{*}$ is Gaussian and looks, apart from the peak position, the same as the likelihood (20) for one given data set $\mathbf{f}$.

To keep things as simple as possible, we assume that there exist (small) integers $n_{\beta}$ for each of the $Z$ pulling velocities $v_{\beta}$ so that $n_{\beta} / n_{\gamma}=\rho_{\beta} / \rho_{\gamma}$. Denoting by $n$ the sum of all $n_{\beta}$, the total number of rupture forces can be written as $N=N^{\prime} n$ and the set of $N$ rupture forces $\mathbf{f}$ can be divided into $N^{\prime}$ subsets $\mathbf{f}^{k}=\left\{f_{i, \beta}^{k}\right\}$ where for fixed $k$, $f_{i, \beta}^{k}$ is one out of the $n_{\beta}$ rupture forces sampled at pulling velocity $v_{\beta}$.

Having introduced this notation, we define $N^{\prime}$ new random variables

$$
\mathbf{X}^{k}=\mathbf{X}^{k}\left(\mathbf{f}^{k}\right)=\frac{1}{n} \sum_{\beta=1}^{Z} \sum_{i=1}^{n_{\beta}} H^{-1} \partial / \partial \boldsymbol{\mu} \ln p_{1}\left(f_{i, \beta}^{k} \mid \boldsymbol{\mu}_{0}, v_{\beta}\right) .
$$

We know already from our discussion in section IIIB that these random variables have an expectation value zero and a covariance matrix

$$
\left\langle\mathbf{X} \mathbf{X}^{\dagger}\right\rangle=\frac{1}{n} H^{-1} .
$$

Then, from the central limit theorem [34] it follows that

$$
\boldsymbol{\mu}^{*}-\boldsymbol{\mu}_{0}=\frac{1}{N^{\prime}} \sum_{k=1}^{N^{\prime}} \mathbf{X}^{k}
$$

is Gaussian distributed with mean zero and covariance matrix

$$
\left\langle\left[\boldsymbol{\mu}^{*}-\boldsymbol{\mu}_{0}\right]\left[\boldsymbol{\mu}^{*}-\boldsymbol{\mu}_{0}\right]^{\dagger}\right\rangle=\frac{1}{n N^{\prime}} H^{-1}=\frac{1}{N} H^{-1} .
$$

\section{APPENDIX B: STATISTICAL UNCERTAINTIES FOR BELL'S MODEL}

In order to determine the covariance matrix of the maximum likelihood estimate for the basic model, in section IVA the quantity $s(\boldsymbol{\mu})$ as defined in (15) had to be calculated. Details of this calculation are given below.

We first calculate

$$
\begin{aligned}
& E(\alpha):=\left\langle e^{\alpha f}\right\rangle_{1}=\int_{0}^{\infty} d f e^{\alpha f} p_{1}\left(f \mid \boldsymbol{\mu}_{0}, v\right) \\
& =\int_{0}^{\infty} d f e^{\alpha f} \frac{e^{\lambda_{0}+\alpha_{0} f}}{r} \exp \left(-\frac{e^{\lambda_{0}}}{r} \frac{e^{\alpha_{0} f}-1}{\alpha_{0}}\right)
\end{aligned}
$$

for an arbitrary loading rate $r=\kappa v$ and $\alpha>-\alpha_{0}$. It is convenient to rewrite equation (B1) using the dimensionless quantities $\tau:=e^{\lambda_{0}} /\left(r \alpha_{0}\right)$ and $\eta=\alpha / \alpha_{0}$ and to substitute $t=\tau \exp \left(\alpha_{0} f\right)$ yielding:

$$
E(\alpha)=\tau^{-\eta} e^{\tau} \int_{\tau}^{\infty} d t t^{\eta} e^{-t} .
$$

Using that $\tau \ll 1$ in typical AFM pulling experiments, we derive at

$$
E(\alpha)=\tau^{-\eta} \Gamma(\eta+1)+\mathcal{O}(\tau),
$$

where $\Gamma(\cdot)$ denotes the Gamma function. Equation (B3) directly gives the expected rupture force:

$$
\langle f\rangle_{1}=\frac{\partial}{\partial \alpha} E(\alpha=0)=-\frac{1}{\alpha_{0}}(\mathcal{C}+\ln \tau)+\mathcal{O}(\tau)
$$

with $\mathcal{C} \approx 0.577$ the Euler constant. Using equations $\underline{B} 3$ B4) and the linearity of the expectation value we obtain:

$$
\begin{aligned}
& -\left\langle\ln p_{1}(f \mid \boldsymbol{\mu}, r)\right\rangle_{1}= \\
& -\lambda+\ln (\kappa v)+\eta(\mathcal{C}+\ln \tau)+\frac{e^{\lambda}}{\kappa v \alpha} \frac{1}{\tau^{\eta}} \Gamma(\eta+1)
\end{aligned}
$$

Finally, equation (B5) together with the definition of the quantity $s(\boldsymbol{\mu})$ yields the desired result (30).

\section{APPENDIX C: OPTIMAL BIN-WIDTH FOR HISTOGRAMS}

Let $p_{1}$ be a probability density function with two continuous and bounded derivatives. For a sample of size $N$ the histogram estimate $\hat{p}_{1}$ of $p_{1}$ is defined as

$$
\hat{p}_{1}(f)=\frac{\Lambda_{N}(f)}{N h_{N}},
$$

with $\Lambda_{N}(f)$ the number of values falling into the bin of width $h_{N}$ around $f$. Then one can show [36, 37] that for large sample sizes the integrated mean squared error

$$
I M S E=\int d f\left\langle\left(\hat{p}_{1}(f)-p_{1}(f)\right)^{2}\right\rangle_{1}
$$

considered as a function of the bin-width is minimized by

$$
h_{N}^{*}=\left(\frac{6}{\int d f\left(p_{1}^{\prime}(f)\right)^{2}}\right)^{1 / 3} N^{-1 / 3} .
$$

Following the same lines as in Appendix $\mathrm{B}$ we obtain for the Bell model:

$$
\int d f\left(p_{1}^{\prime}(f \mid \boldsymbol{\mu}, v)\right)^{2}=\frac{1}{8} \alpha^{3}\left(1+\mathcal{O}\left(\frac{2 e^{\lambda}}{\kappa v \alpha}\right)\right) .
$$

Inserting (C4) into (C3) yields the optimal bin-width:

$$
h_{N}^{*} \approx 3.63 \frac{1}{\alpha N^{1 / 3}} .
$$

It should be noted that in the limit $\frac{2 e^{\lambda}}{\kappa v \alpha} \ll 1$, the optimal bin-width depends solely on the sample size $N$ and on 
the parameter $\alpha$ which determines the width of the distribution, but neither on the force-free dissociation rate $k_{0}=\exp (\lambda)$ nor on the pulling velocity $v$.

If one wishes to determine the optimal bin-width prior to parameter estimation, one may make use of the well known result $\left\langle\left(f-\langle f\rangle_{1}\right)^{2}\right\rangle_{1} \approx \pi^{2} /\left(6 \alpha^{2}\right)$ and choose

$$
h_{N}=2.83 s_{N} N^{-1 / 3}
$$

where $s_{N}$ denotes the standard deviation of the measured rupture forces. This estimate is pretty close to the optimal bin-width $h_{N, \text { Gauss }} \approx 3.49 s_{N} N^{-1 / 3}$ of a Gaussian distribution [36].
[1] R. Merkel, Phys. Rep. 346, 343 (2001).

[2] E. Evans, Annu. Rev. Biophys. Biomol. Struct. 30, 105 (2001).

[3] P. Hinterdorfer, W. Baumgartner, H.J. Gruber, K. Schilcher, and H. Schindler, Proc. Natl. Acad. Sci. USA 93, 3477 (1996).

[4] F.W. Bartels, M. McIntosh, A. Fuhrmann, Ch. Metzendorf, P. Plattner, N. Sewald, D. Anselmetti, R. Ros, and A. Becker, Biophys. J. 92, 4391 (2007).

[5] W. Wernsdorfer, E. Bonet Orozco, K. Hasselbach, A. Benoit, B. Barbara, N. Demoncy, A. Loiseau, H. Pascard, and D. Mailly, Phys. Rev. Lett. 78, 1791 (1997).

[6] E. Riedo, E. Gnecco, R. Bennewitz, E. Meyer, and H. Brune, Phys. Rev. Lett. 91, 084502 (2003).

[7] A. Schirmeisen, L. Jansen, and H. Fuchs, Phys. Rev. B 71, 245403 (2005).

[8] M. Evstigneev, A. Schirmeisen, L. Jansen, H. Fuchs, and P. Reimann, Phys. Rev. Lett. 97, 240601 (2006).

[9] J. Kurkijärvi, Phys. Rev. B 6, 832 (1972).

[10] T.A. Fulton and L.N. Dunkleberger, Phys. Rev. B 9, 4760 (1974)

[11] G.I. Bell, Science 200, 618 (1978).

[12] E. Evans and K. Ritchie, Biophys. J. 72, 1541 (1997).

[13] P. Hänggi, P. Talkner, and M. Borkovec, Rev. Mod. Phys. 62, 251 (1990).

[14] M. Rief, J.M. Fernandez, and H.E. Gaub, Phys. Rev. Lett. 81, 4764 (1998)

[15] R. Merkel et al. Nature 397, 50 (1999).

[16] T. Strunz et al., Biophys. J. 79, 1206 (2000).

[17] B. Heymann and H. Grubmüller, Phys. Rev. Lett. 84, 6126 (2000).

[18] U. Seifert, Phys. Rev. Lett. 84, 2750 (2000).

[19] B. Isralewitz, M. Gao, and K. Schulten, Curr. Opin.
Struct. Biol. 11, 224 (2001).

[20] D. Bartolo, I. Derényi, and A. Ajdari, Phys. Rev. E 65, 051910 (2002).

[21] N. Nguyen-Duong, K.W. Koch, and R. Merkel, Europhys. Lett. 61, 845 (2003).

[22] O.K. Dudko, A.E. Filippov, J. Klafter, and M. Urbakh, Proc. Natl. Acad. Sci. USA 100, 11378 (2003);

[23] G. Hummer and A. Szabo, Biophys. J. 85, 5 (2003).

[24] C.E. Maloney and D.J. Lacks, Phys. Rev. E 73, 061106 (2006).

[25] O.K. Dudko, G. Hummer, and A. Szabo, Phys. Rev. Lett. 96, 108101 (2006).

[26] F. Hanke and H.J. Kreuzer, Phys. Rev. E 74, 031909 (2006).

[27] J. Husson and F. Pincet, Phys. Rev. E 77, 026108 (2008).

[28] S. Izrailev, S. Stepaniants, M. Balsera, Y. Oono, and K. Schulten, Biophys. J. 72, 1568 (1997).

[29] S. Getfert and P. Reimann, Phys. Rev. E 76, 052901 (2007).

[30] O.K. Dudko, J. Mathé, A. Szabo, A. Meller, and G. Hummer, Biophys. J. 92, 4188 (2007).

[31] G. D'Agostini, Rep. Prog. Phys 66, 1383 (2003).

[32] V. Dose, Rep. Prog. Phys 66, 1421 (2003).

[33] T.M. Cover and J.A. Thomas, Elements of Information Theory, Wiley, New York, 1991.

[34] H. Cramér, Mathematical Methods of Statistics, Princeton University Press, Princeton 1946.

[35] M. Evstigneev and P. Reimann, Phys. Rev. E 68, 045103(R) (2003) .

[36] D.W. Scott, Biometrika 66, 605 (1979).

[37] R. Mats, Scand. J. Statist. 9, 65 (1982). 